\title{
The associations of TMJ pain and bone characteristics on the activities of daily living
}

\author{
Oksana Jagur ${ }^{1}$, Mart Kull², Edvitar Leibur ${ }^{1,2}$, Riina Kallikorm² ${ }^{2}$, Margus Lember ${ }^{2}$, Ülle Voog-Oras ${ }^{1}$ \\ ${ }^{1}$ Department of Stomatology, Tartu University Hospital, University of Tartu, Tartu, Estonia \\ ${ }^{2}$ Department of Internal Medicine, University of Tartu, Tartu, Estonia \\ Email: oksana.jagur@kliinikum.ee
}

Received 28 June 2012; revised 29 July 2012; accepted 6 August 2012

\begin{abstract}
The aim of this investigation was to analyze the associations of TMJ pain/discomfort and bone characteristics on the activities of daily living in a population based study. Methods: The study included 95 randomly selected participants. The impact of temporomandibular joint pain/discomfort on the daily activeties was assessed by using the daily living scale by List and Helkimo (1995). The levels of serum type I collagen telopeptide fragments (P1NP), C-telopeptide crosslaps of type I collagen (CTX-1), and vitamin D (25(OH)D) were analysed. The Mann-Whitney U-test, Spearman rank correlation coefficent $\left(r_{s}\right)$ were used for statistical analyses. Results: Activities of daily living were influenced by temporomandibular joint pain/ discomfort to varying degrees. The degree of pain/ discomfort during eating was correlated with the level of C-telopeptide crosslaps of type I collagen $(p=0.02)$ and type 1 collagen telopeptide fragments $(p=0.04)$. The females in the study had significantly lower vitamin D levels compared to males $(p=0.04)$. Conclusions: This population based study indicates that TMJ pain/discomfort exerts a significant negative influence on activities of daily living and is related to the biochemical markers of bone turnover and 25(OH)D level. Several functions of daily activities such as social life, exercising and eating are the most disturbed.
\end{abstract}

Keywords: Temporomandibular Joint; Activities of Daily Living; Bone Characteristics; Pain Assessment; Vitamin D

\section{INTRODUCTION}

The temporomandibular joint pain is commonly accompanied by stiffness, sounds and functional limitations contributing to a decreased quality of life, and thus having a significant negative impact on activities of daily living. According to the survey, pain prevented $42 \%$ of subjects from working and $63 \%$ of subjects from carrying out their daily activities [1]. In a study of general population it was reported that $14 \%$ of the American population suffered from chronic musculoskeletal pain [2]. In a Swedish study $40 \%$ of adult population reported pain that afflicted them to "quite high degree" for more than 6 months [3]. Chronic craniofacial pain conditions with a prevalence of approximately $10 \%-15 \%$ are increasing in the adult population [3-6]. A human experimental pain model has been used to estimate jaw-motor functions, offering for the understanding of these functions in relation to painful temporomandibular dysfunction conditions [7]. Chronic pain may be nociceptive, neuropathic, ischemic, and visceral or exhibit a combination of different etiologies. Nociceptive pain may result from the stimulation of nociceptors at the nerve endings characteristic in case of temporomandibular joint (TMJ) disorders. Pain negatively affects the patients' psychosocial and physical well-being. A patient's quality of life can also be adversely influenced by the interplay of a peripheral nociceptive source in a muscle, a faulty central nervous system component and decreased coping ability. It is widely accepted that a complex interaction of variable intrinsic and extrinsic factors can induce persistent orofacial muscle pain and dysfunction [8].

Many patients experience sleep disturbances, disrupttions, decreased physical functioning, interference with activities of daily living, decreased productivity, increased anxiety and depression, and decreased quality of life [1]. These clinical symptoms and signs in the TMJ produce an impact not only on specific aspects of daily living but also on the patients'social life in general [9].

Assessment of individual daily activities level is important in the evaluation of TMJ disorders. There are several rating scales for assessing patients' TMJ functions and for describing the details of their disability and how their current status is reflected in their day to day activities.

The term "activities of daily living" (ADL) has been used to denote activities undertaken as part of a person's 
daily functions. These activities are often divided into physical activities of daily living (ADL) and instrumental activities of daily living (IADL); [10].

The first reference to activities of daily living was made by Sheldon in the Journal of Health and Physical Education in 1935 [11].

The ADL scale by Katz S. et al. [12] was primarily designed to measure the ability to carry out basic activeties necessary for daily living. It has been validated and modified for specific use in patients with TMJ disorders. There are several rating scales for measuring the severity of chronic pain and its effects in terms of interference with ADL. A rating scale has been elaborated for measuring the $\mathrm{ADL}$ of patients with craniomandibular disorders [13]. The Jaw Pain and Function Questionnaire, a self rating scale, was originally published by Clark et al. [14]. The questionnaire consists of a visual analogue scale (VAS), 8 questions about pain, 5 about jaw dysfunction, and 18 about daily activities. The verbal items are scored from zero to four points; the range of time being evaluated covers the previous week.

The VAS was initially used in psychology by Freyd and others in the early 1900s and was elaborated in rheumatology through a series of investigations by Huskisson et al. in the late 1970s [15].

The Research Diagnostic Criteria for Temporomandibular Disorders have been shown to be a reliable way of characterising bone changes and are widely used to diagnose and assess temporomandibular disorders [16-19].

Several markers, as serum type 1 collagen telopeptide fragments (P1NP), C telopeptide crosslaps of type 1 collagen (CTX-1) and C-terminal telopeptide of type 1 collagen (1CTP) and procollagen type $1 \mathrm{~N}$-terminal propeptide play an important role in the bone behaviour. They are currently considered highly sensitive markers of bone metabolism and its significant relation with radiographic changes in the TMJ have been found [20-22].

CTX-1 is generated by cathepsin $\mathrm{K}$, being the key of osteoclastic enzyme for systemic bone resorption. In contrast, 1CTP is generated by matrix metalloproteinases whose activity plays an important role in collagen degradation associated with systemic inflammatory diseases $[23,24]$.

The measurement of serum procollagen type $1 \mathrm{~N}$-terminal propeptide level appears a sensitive marker of bone formation rate in osteoporosis [21,25]. Vitamin D $(25(\mathrm{OH}) \mathrm{D})$ plays a role in calcium and bone metabolism, inhibiting cytokine production and proliferation of various tissues $[21,26]$. In a study of community-dwelling older adults it was found that vitamin D deficiency is common and is associated with poor physical performance, lower muscle strength, as well as prevalent mobility and ADL disability [26]. Biochemical markers of bone turnover can be used to predict individual bone loss.
They may help to alert patients to the risk of pathologies in the TMJ [27].

Thus studies which evaluate the above mentioned contributing factors to explore the relationship between TMJ pain, certain bone characteristics and ADL among general population are justified.

The hypothesis for this investigation was to find whether TMJ pain/discomfort and biochemical markers of bone turnover can influence the activities of daily living depending on the gender.

The aim of this investigation was to analyze the associations of TMJ pain/discomfort and bone characteristics on the activities of daily living in a population based study.

\section{MATERIAL AND METHODS}

The study was approved by the Ethical Committee of the Faculty of Medicine, University of Tartu (protocol no. 140/18, 2005). All participants gave their informed consent before the start of the study.

The study was conducted in 2006 in the municipality of Väike-Maarja (Estonia). A group of randomly selected participants $(n=103)$ consisting of 61 females and 42 males (aged 26 - 70) from the patient register of a local family practitioner were invited to participate, and of those invited, 95 (92\%) persons took part in the study.

All participants were asked to evaluate the influence on daily activities of pain/discomfort in the TMJ. A rating scale based on methods elaborated in medical and behavioural science and modified by List and Helkimo was used [13]. The questions in the scale were translated from English into Estonian by one of the authors [9]. A modification of the rating scale was used where one of the questions (question 10) in the original scale was excluded since it was proved inadequate [13]. The scale was defined as 0 (activity without any pain/discomfort in the TMJ) to 10 (activity impossible due to pain/discomfort in the TMJ). The participants were asked to mark the number that best described their present ability to perform each activity considering their pain/discomfort from TMJ. The questions used in the study were (English original versions): If you feel pain/discomfort in the area of TMJ are you able to:

- Socialize with family and close friends? (ADL 1);

- Perform daily work? (ADL 2);

- Perform daily household chores (preparing meals, cleaning, taking care of small children)? (ADL 3);

- Sit in a company or participate in other social activities (e.g. parties)? (ADL 4);

- Exercise (walk, bicycle, jog, etc)? (ADL 5);

- Perform hobbies (read, fish, knit, play an instrument)? (ADL 6);

- Sleep at night? (ADL 7);

- Concentrate? (ADL 8); 
- Eat (chew, swallow)? (ADL 9);

- Talk (laugh, sing)? (ADL 10);

- Yawn, open mouth wide? (ADL 11);

- How much does the pain/discomfort affect your daily activities? (ADL 12).

Quantitative changes in bone turnover were assessed by the measurement of serum levels of biochemical markers as follows: 25(OH)D, CTX-1 and P1NP.

All samples were taken in the morning (after an overnight fast) between 8 a.m. and noon using pre-cooled tubes. Serum was separated and the samples were stored at $-20^{\circ} \mathrm{C}$ until analyzed. All samples were analyzed simultaneously and in duplicate to minimize inter-assay variations. Analyses were performed by the United Laboratories of the Tartu University Hospital.

The 25(OH)D level (collected in winter) in the serum was measured by radioimmunosorbent assay produced by DiaSorin (Stillwater, Minnesota, USA). The intra and inter-assay CVs were $4.1 \%$ and $5.7 \%$ respectively. For group discrimination, we used $25 \mathrm{nmol} / \mathrm{L}$ as the critical value for deficiency and $50 \mathrm{nmol} / \mathrm{L}$ as the cut-off value for insufficiency. A value of $75 \mathrm{nmol} / \mathrm{L}$ was considered the optimal 25(OH)D level [23]. P1NP was determined by Elecsys 1010/2010 total P1NP serum kit (Roche Diagnostics, Mannheim, Germany), which employs the electrochemiluminescence immunoassay (ECLIA) technique. Bone formation marker P1NP reference range for premenopausal women is 15.1 - $58.6 \mathrm{ng} / \mathrm{mL}$, for postmenopausal women $16.3-73.9 \mathrm{ng} / \mathrm{mL}$ and for men aged 51 to 70 years $<36.4 \mathrm{ng} / \mathrm{mL}$. CTX-1 was determined by Elecsys 1010/2010 $\beta$-CrossLaps/serum kit (Roche Diagnostics, Mannheim, Germany) whose sensitivity of assay is $0.01 \mathrm{ng} / \mathrm{mL}$. The mean (SD) and mean $+2 \mathrm{SD}$ figures are as follows: for premenopausal women $0.025-0.573$ $\mathrm{ng} / \mathrm{mL}$; for postmenopausal women $0.104-1.008 \mathrm{ng} / \mathrm{mL}$; for men aged 31 to 51 years $0.016-0.584 \mathrm{ng} / \mathrm{mL}$; for men aged 51 to 71 years $0.096-0.704 \mathrm{ng} / \mathrm{mL}$; and for men older than 71 years $0.066-0.854 \mathrm{ng} / \mathrm{mL}$.

The variables were tested for differences between male and female groups with the Mann-Whitney U-test. The significance of the correlations was tested by Spearman rank correlation coefficient $\left(\mathrm{r}_{\mathrm{s}}\right)$. A significance level of less than 0.05 was considered as significant.

\section{RESULTS}

The impact of TMJ pain/dyscomfort on daily living activities in this study was found to vary between activities and between the sexes. The influence from quantitative changes in bone turnover appeared to play a significant role on activities on daily living.

\subsection{Relation between Males and Females}

The subgroup of females was characterised by highly significant correlations with the following activities of daily living: social life (ADL $1 ; p=0.015$ ), perform daily work (ADL $2 ; p=0.024)$, perform daily household chores (ADL 3; $p=0.021)$, exercise (ADL 5; $\mathrm{p}=0.003)$, perform hobbies (ADL 6; $\mathrm{p}=0.023$ ) and yawn, open mouth wide (ADL 11; $\mathrm{p}=0.009$ ).

The females had significantly lower 25(OH)D levels compared to males $(\mathrm{p}=0.04)$.

\subsection{Relation between ADL and Bone Characteristics}

The median levels of bone characteristics are given in Table 1.

Statistically significant differences were found between ADL data and bone status.

The distribution of significant results between ADL data and bone characteristics is shown in Figure 1. The correlations between ADL data and the serum levels of bone characteristics (CTX-1, P1NP and 25(OH)D) were found (Table 2).

\subsection{Relations between ADL, TMJ Pain, Age and Gender}

The participants'age was not significantly correlated to any ADL questions. The relations between ADL and the sexes are presented in Table 3 . The median value of TMJ

Table 1. Serum levels of bone characteristics.

\begin{tabular}{cccc}
\hline Bone characteristics & Gender & Median & IQR \\
\hline $25(\mathrm{OH}) \mathrm{D}$ & & & \\
& $\mathrm{F}$ & 58.5 & 22.3 \\
$\mathrm{P} 1 \mathrm{NP}$ & $\mathrm{M}$ & 54.5 & 23.3 \\
& $\mathrm{~F}$ & 40.0 & 24.5 \\
& $\mathrm{M}$ & 42.5 & 22.2 \\
CTX-1 & & & \\
& $\mathrm{F}$ & 0.3 & 0.2 \\
& $\mathrm{M}$ & 0.3 & 0.2 \\
\hline
\end{tabular}

IQR $=$ interquartile range, Gender $(\mathrm{M}=$ male, $\mathrm{F}=$ female $), 25(\mathrm{OH}) \mathrm{D}=$ Vitamin D in nmol/L, P1NP = Procollagen-1N-collagen in ng/L, CTX-1 = C-telopeptide of type I collagen in $\mathrm{ng} / \mathrm{mL}$.

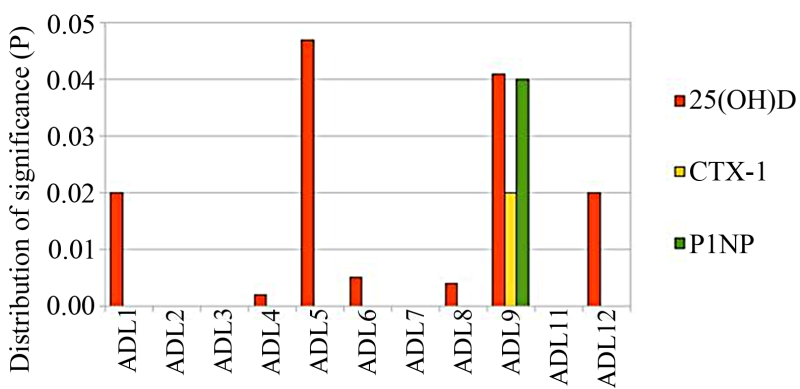

Relationship between bone characteristics and activities of daily living.

Figure 1. Distribution of significance between ADL data and bone characteristics. 
Table 2. Correlations between ADL questions and bone characteristics.

\begin{tabular}{|c|c|c|c|c|c|c|c|c|}
\hline \multicolumn{3}{|c|}{ TMJ pain episodes } & \multicolumn{2}{|c|}{$25(\mathrm{OH}) \mathrm{D}$} & \multicolumn{2}{|c|}{ CTX-1 } & \multicolumn{2}{|c|}{ P1NP } \\
\hline & $\mathrm{r}_{\mathrm{s}}$ & $\mathrm{p}$ & $\mathrm{r}_{\mathrm{s}}$ & $\mathrm{p}$ & $r_{s}$ & $\mathrm{p}$ & $r_{s}$ & $\mathrm{p}$ \\
\hline ADL1 & 0.41 & $<0.001$ & -0.26 & 0.017 & NS & NS & NS & NS \\
\hline ADL2 & 0.52 & $<0.001$ & NS & NS & NS & NS & NS & NS \\
\hline ADL3 & 0.51 & $<0.001$ & NS & NS & NS & NS & NS & NS \\
\hline ADL4 & 0.41 & $<0.001$ & -0.32 & 0.002 & NS & NS & NS & NS \\
\hline ADL5 & 0.50 & $<0.001$ & -0.21 & 0.047 & NS & NS & NS & NS \\
\hline ADL6 & 0.40 & $<0.001$ & -0.30 & 0.005 & NS & NS & NS & NS \\
\hline ADL7 & 0.43 & $<0.001$ & NS & NS & NS & NS & NS & NS \\
\hline ADL8 & 0.48 & $<0.001$ & -0.31 & 0.004 & NS & NS & NS & NS \\
\hline ADL9 & 0.43 & $<0.001$ & -0.22 & 0.041 & -0.24 & 0.020 & -0.20 & 0.051 \\
\hline ADL11 & 0.63 & $<0.001$ & NS & NS & NS & NS & NS & NS \\
\hline ADL12 & 0.51 & $<0.001$ & -0.26 & 0.017 & NS & NS & NS & NS \\
\hline
\end{tabular}

TMJ pain episodes $=$ questions about having pain ever in the TMJ area, $\mathrm{r}_{\mathrm{s}}=$ Spearman rank correlation coefficient, $\mathrm{P}=\mathrm{a}$ significance level of less than 0.05 was considered as significant, $25(\mathrm{OH}) \mathrm{D}=$ Vitamin $\mathrm{D}$ in nmol/L, CTX-1 = C-telopeptide of type I collagen in ng/mL, P1NP $=$ Procollagen-1N-collagen in ng/L, NS $=$ non-significant correlation .

Table 3. The influence of temporomandibular joint pain/discomfort on activities of daily living.

\begin{tabular}{|c|c|c|c|c|c|c|}
\hline ADL questions & Median & IQR & $\%$ pos. & Median & IQR & $\%$ pos. \\
\hline & & Male & & & Female & \\
\hline ADL1 & 0 & 4 & 45 & 3 & 5 & 74 \\
\hline ADL2 & 0 & 3 & 37 & 2 & 4 & 68 \\
\hline ADL3 & 0 & 3 & 37 & 2 & 4 & 61 \\
\hline ADL4 & 1 & 5 & 50 & 4 & 6 & 68 \\
\hline ADL5 & 0 & 3 & 37 & 2 & 5 & 74 \\
\hline ADL6 & 0 & 3 & 45 & 3 & 5 & 67 \\
\hline ADL7 & 1 & 5 & 50 & 3 & 6 & 67 \\
\hline ADL8 & 2 & 5 & 53 & 3 & 5 & 74 \\
\hline ADL9 & 3 & 7 & 68 & 3 & 4 & 77 \\
\hline ADL11 & 0 & 3 & 50 & 3 & 6 & 68 \\
\hline ADL12 & 1 & 4 & 58 & 3 & 4 & 75 \\
\hline
\end{tabular}

$\mathrm{IQR}=$ interquartile range, ADL scale: 0 - 12 where $0=$ activity without any pain/discomfort at all and $12=$ activity impossible due to pain/discomfort, $\%$ pos: percentage of observations exceeding zero.

pain was $76.9 \%$ in female cases (IQR 5) and $53.2 \%$ in male cases (IQR 4.2). The women were more affected by pain/discomfort in TMJ than men. A correlation between TMJ pain/discomfort and ADL was found in all ADL questions. TMJ pain in general was correlated with all ADL items $\left(r_{s}=0.53, p<0.01\right)$. The impact of TMJ pain/dyscomfort was the greatest during eating (ADL 9; $68 \%$ and $77 \%$ respectively in the male and female subgroup) and smallest in performing daily household chores (ADL $3 ; 37 \%$ and $61 \%$ respectively in the male and female subgroup). Subjects who experienced a higher degree of TMJ pain/discomfort reported a significant negative impact on activities of daily living.

\section{DISCUSSION}

The population-based random study reported in this article demonstrates that TMJ pain/discomfort has a significant negative impact on activities of daily living. We found correlations between TMJ pain/discomfort and activities of daily living throughout ADL data. All correlations were significant, especially those with eating, yawning, opening the mouth wide, performing daily work and sleeping at night. Similar results have been reported by other investigators $[9,28,29]$. The degree of influence on eating, yawning and mouth opening could easily be explained by TMJ pain, escpecially since all 
ADL questions were correlated with previous TMJ pain episodes. It was found that a relatively high median pain level influenced the performance of daily work. The influence on sleep at night might also be due to TMJ problems since disc dislocation and the corresponding pain sensation can easily be provoked by sleeping in a prone position. The relatively minor influence of TMJ pain on performing daily household chores in both gender groups was unexpected. It may perhaps be explained by the individual ability to tolerate pain or ascribed to increased pain coping ability. The median TMJ pain value in the male as well as female group was relatively high if we take into account the fact that our sample consisted of voluntary participants. It shows a relatively high prevalence of TMJ problems in population based investigation [30]. Our findings (58 females and 37 males, mean age 55 ) indicated that middle-aged women have more complaints than middle-aged men. Especially complaints were represented in daily work, social activities, hobbies, physical exercises. Concentration difficulties were reported more often by women than by men. Women were also more affected by pain. This matches the findings of other studies which also concluded that the incidence of chronic craniofacial pain conditions among women is 1 , 5 to 2 times higher than among men [31,32].

With regard to biological factors, quantitative as well as qualitative differences in endogenous pain inhibitory systems have been implicated, as well as an influence of gonadal hormones. Psychosocial factors such as sex role beliefs, pain coping strategies, and pain related expectancies may also contribute to the differences [33].

TMJ pain is frequently accompanied by psychological distress, notably depression and somatization, and can also be associated with psychosocial disability, including pain-related interference with habitual work, home, and interpersonal activities and an extensive use of health care services [34].

The participants' age was not significantly correlated to any ADL questions. It might be explained due to the relatively high median age in this study (55 years). It has been reported that changes in the TMJ due to remodeling or arthritis mainly appear after the age of 45 [35].

In our study the females had significantly lower $25(\mathrm{OH}) \mathrm{D}$ level compared to the males. Female participants felt more able to engage in social life, perform daily work, perform daily household chores, exercise, go in for hobbies, yawn and open the mouth wide. Vitamin $\mathrm{D}$ insufficiency does not seem to be a serious problem among the active elderly, yet elderly people with a low level of activities of daily living seem to run a very high risk of vitamin D insufficiency [36].

It is obvious that persons with TMJ pain experience a (substantial) psychological impact and disruption of their social and recreational activities [37]. The impact of TMJ pain/discomfort on ADL in this study was found to vary between activities and individuals. Work disability results from a complex interactions of demographic variables and backround factors.

ADL scale results may change the process of treatment. This questionnaire is very simple and it can be recommended for future clinical trials in patients with TMJ disorders [28].

Pain is a personal experience and information about a patient's pain can be obtained only from the person. An ADL questionnaire is a very easy method for pain assessment but self-reporting scales have been found a more or less reliable reflection on actual ADL ability only in healthy or very mildly affected individuals [10].

Patient questionnaires do not replace further consultation of the patient's medical history, physical examinations, laboratory investigations or other tests, and must be interpreted by the physician for correct patient management similarly to laboratory tests and other quantitative data $[15,38]$. While advances in therapy require laboratory, radiological and other tests, patient questionnaires provide optimal scientific data for clinical use.

Only a few systematic reviews have appeared with respect to daily activities or quality of life in the management of TMJ disorders. It was found in those reviews that pain and clinical features as primary outcome variables in the TMJ have been estimated are to be taken into account $[37,39]$ while a few systemic reviewers reported the role of psychological status, daily activities, or quality of life $[9,19]$.

As vitamin D status is considered a determinant of bone health the assessment of its level is important. It has been demonstrated that $25(\mathrm{OH}) \mathrm{D}$ levels are low all year round in Estonia [21]. In our study the mean serum $25(\mathrm{OH}) \mathrm{D}$ level in females was $58.5 \mathrm{nmol} / \mathrm{L}$, and in males $54.5 \mathrm{nmol} / \mathrm{L}$. Subjects with lower $25(\mathrm{OH}) \mathrm{D}$ values experience difficulties in performing physical exercises, engaging in hobbies, they have problems with eating, participating in static social gatherings or other social activities. These persons' social life is disrupted. A low 25(OH)D level can also predict TMJ bone destruction. Therefore, the destruction of mineralized tissues in the TMJ might be slowed or counteracted by correcting the patient's 25(OH)D levels [22].

Not much clinical research has focused on the specific effects of vitamin D deficiency on jaw pain. Vitamin D deficiency can result in a predisposition to TMJ disorders and musculoskeletal pain [40]. Based on our results, additional vitamin $\mathrm{D}$ consumption might be suggested to avoid TMJ disorders. We may assume that there is a possible relationship between low 25(OH)D levels, TMJ pain/discomfort and the quality of life.

P1NP affects osteoblast activity, thus enhancing bone formation. It will result in an elevated P1NP concentra- 
tion and is useful in monitoring the response to bone formation. Low P1NP values may predict various bone diseases. In our data the P1NP level remained in reference range (the median serum P1NP level in females and males respectively was $40.0 \mathrm{nmol} / \mathrm{L}$ and $42.5 \mathrm{nmol} / \mathrm{L}$ ). A statistically significant difference was found between the patients' ADL data and P1NP levels. P1NP level depends on many factors such as diet, the level of physical activeity, season, etc. [21].

Subjects with normal P1NP levels tend to be more active physically and socially. In our study CTX-1 correlated positively with P1NP and negatively with 25(OH)D. Previous research has shown that subjects with an increased level of bone metabolism markers CTX-1 and $\mathrm{P} 1 \mathrm{NP}$ are likely to have a 25(OH)D insufficiency [21].

We found that subjects with increased levels of P1NP, CTX-1 have less TMJ pain/discomfort. Increased levels of CTX-1 would probably cause an immediate increase of P1NP which is known as a sensitive marker of bone formation. It could be explained by a shift in the equilibrium between bone resorption and formation markers in healthy individuals. Due to that the contribution of different pain modulating mediators such as serotonin (5HT) etc. in the TMJ area probably remains modest.

The clinical significance of the results from this study is that the determination of P1NP, CTX-1 and 25(OH)D serum levels in order to estimate bone status of the TMJ is confirmed.

This population based study indicates that pain/discomfort from the TMJ is influenced by the serum levels of biochemical markers and 25(OH)D.

\section{CONCLUSION}

This population based study indicates that TMJ pain/ discomfort exerts a significant negative influence on activities of daily living and is related to the biochemical markers of bone turnover and 25(OH)D level. Several functions of daily activities such as social life, exercising and eating are the most disturbed.

\section{ACKNOWLEDGEMENTS}

The study was financially supported by the targeted funding project SF 0180081 s07 and by grant 6591 of the Estonian Science Foundation.

The authors would like to thank their colleagues form the Department of Internal Medicine and United Laboratories of Tartu University Hospital.

None of the authors is aware of any financial or personal relationships with people or organizations which might have inappropriately influenced this study.

\section{REFERENCES}

[1] Katz, W.A. and Barkin, R.L. (2010) Dilemmas in chronic/ persistent pain management. Disease-A-Month, 56, 233 250. doi:10.1016/j.disamonth.2009.12.006

[2] Magni, G., Caldieron, C., Rigatti-Luchini, S., et al. (1990) Chronic musculoskeletal pain and depressive symptoms in the general population. An analysis of the 1st national health and nutrition examination survey data. Pain, 43, 299-307. doi:10.1016/0304-3959(90)90027-B

[3] Brattberg, G., Thorslund, M. and Wikman, A. (1989) The prevalence of pain in a general population. The results of a postal survey in a county of Sweden. Pain, 37, 215-222. doi:10.1016/0304-3959(89)90133-4

[4] Dworkin, S.F., Huggins, K.H., LeResche, L., et al. (1990) Epidemiology of signs and symptoms in temporomandibular disorders: Clinical signs in cases and controls. Journal of the American Dental Association, 120, 273-281.

[5] LeResche, L. (1997) Epidemiology of temporoman-dibular disorders: Implications for the investigation of etiologic factors. Critical Reviewers in Oral Biology \& Medicine, 8, 291-305. doi:10.1177/10454411970080030401

[6] John, M.T., Dworkin, S.F. and Mancl, L.A. (2005) Reliability of clinical temporomandibular disorder diagnoses. Pain, 118, 61-69. doi:10.1016/j.pain.2005.07.018

[7] Svensson, P. (2007) What can human experimental pain models teach us about clinical TMD? Archives of Oral Biology, 52, 391-394.

doi:10.1016/j.archoralbio.2006.11.015

[8] Benoliel, R., Svensson, P., Heir, G.M., et al. (2011) Persistent orofacial muscle pain. Oral Disease, 7, 23-41. doi:10.1111/j.1601-0825.2011.01790.x

[9] Voog, Ü., Alstergren, P., Leibur, E., et al. (2003) Impact of temporomandibular joint pain on activities of daily living in patients with rheumatoid arthritis. Acta Odontologica Scandinavica, 61, 278-282. doi:10.1080/00016350310005989

[10] Bucks, S.R. and Haworth, J. (2002) Bristol activities of daily living scale: A critical evaluation. Expert Review of Neurotherapeutics, 2, 669-676. doi:10.1586/14737175.2.5.669

[11] Feinstein, A.R., Josephy, B.R. and Wells, C.K. (1986) Scientific and clinical problems in indexes of functional disability. Annals of Internal Medicine, 105, 413-420.

[12] Katz, S., Ford, A.B., Moskowitz, R.W., et al. (1963) Studies of illness in the aged. The index of ADL: A standardized measure of biological and psychological function. The Journal of the American Medical Association, 185, 914-919. doi:10.1001/jama.1963.03060120024016

[13] List, T. and Helkimo, M. (1995) A scale for measuring the activities of daily living (ADL) of patients with craniomandibular disorders. Swedish Dental Journal, 19, 33-40.

[14] Clark, G.T., Seligman, D.A., Solberg, W.K., et al. (1989) Guidelines for the examination and diagnosis of temporomandibular disorders. Journal of Craniomandibular Disorders, 3, 7-14.

[15] Sokka, T. (2003) Assessment of pain in patients with rheumatic diseases. Best Practise \& Research Clinical Rheumatology, 17, 427-449. doi:10.1016/S1521-6942(03)00020-2 
[16] Von Korff, M., Ormel, J., Keefe, F.J., et al. (1992) Grading the severity of chronic pain. Pain, 50, 133-149. doi:10.1016/0304-3959(92)90154-4

[17] Wahlund, K., List, T. and Dworkin, S.F. (1998) Temporomandibular disorders in children and adolescents: Reliability of questionnaire, clinical examination, and diagnosis. Journal of Orofacial Pain, 12, 42-51.

[18] Schiffman, E.L., Ohrbach, R., Truelove, E.L., et al. (2010) The Research Diagnostic Criteria for Temporomandibular Disorders. V: Methods used to establish and validate revised Axis I diagnostic algorithms. Journal of Orofacial Pain, 24, 63-78.

[19] List, T. and Axelsson, S. (2010) Management of TMD: Evidence from systematic reviews and meta-analyses. Journal of Oral Rehabilitation, 37, 430-451. doi:10.1111/j.1365-2842.2010.02089.x

[20] Garnero, P., Ferreras, M., Karsdal, M.A., et al. (2003) The type I collagen fragments ICTP and CTX reveal distinct enzymatic pathways of bone collagen degradation. Journal of Bone and Mineral Research, 18, 859-867. doi:10.1359/jbmr.2003.18.5.859

[21] Kull Jr., M., Kallikorm, R., Tamm, A., et al. (2009) Seasonal variance of $25-(\mathrm{OH})$ vitamin $\mathrm{D}$ in the general population of Estonia, a Nothern European country. BMC Public Health, 9, 22-33. doi:10.1186/1471-2458-9-22

[22] Jagur, O., Kull, M., Leibur, E., et al. (2011) Relationship between radiographic changes in the temporomandibular joint and bone mineral density: A population based study. Stomatologija, Baltic Dental and Maxillofacial Journal, 13, 42-48.

[23] Chopin, F., Garnero, P., le Henanff, A., et al. (2008) Longterm effects of infliximab on bone and cartilage turnover markers in patients with rheumatoid arthritis. Annals of the Rheumatic Diseases, 67, 353-357. doi:10.1136/ard.2007.076604

[24] Reid, I.R., Davidson, J.S., Wattie, D., et al. (2004) Comparative responses of bone turnover markers to bisphosphonate therapy in Paget's disease of bone. Bone, 35, 224-230. doi:10.1016/j.bone.2004.03.023

[25] Dietrich, T., Joshipura, K.J., Dawson-Hughes, B., et al (2004). Association between serum concentrations of 25-hydroxyvitamin D3 and periodontal disease in the US population. The American Journal of Clinical Nutrition, 80, 108-113.

[26] Houston, D.K., Tooze, J.A., Davis, C.C., et al. (2011) Serum 25-hydroxyvitamin D and physical function in older adults: The cardiovascular health study all stars. Journal of the American Geriatrics Society, 59, 17931801. doi:10.1111/j.1532-5415.2011.03601.x

[27] Vlasiadis, K.Z., Damilakis, J., Velegrakis, G.A., et al. (2008) Relationship between BMD, dental panoramic radiographic findings and biochemical markers of bone turnover in diagnosis of osteoporosis. Maturitas, 59, 226-233. doi:10.1016/j.maturitas.2008.01.006

[28] Undt, G., Murakami, K., Clark, G.T., et al. (2006) Crosscultural adaptation of the JPF-Questionnarie for German speaking patients with functional temporomandibular joint disorders. Journal of Cranio-Maxillofacial Surgery, 34, 226-233. doi:10.1016/j.jcms.2005.12.005

[29] Tjakkes, G.H., Reinders, J.J., Tenvergert, E.M., et al. (2010) TMD pain: The effect on health related quality of life and the influence of pain duration. Health and Quality of Life Outcomes, 8, 46. doi:10.1186/1477-7525-8-46

[30] Chong, D.K. (1995) Measurement of instrumental activities of daily living in stroke. Stroke, 26, 1119-1122. doi:10.1161/01.STR.26.6.1119

[31] Dao, T.T. and LeResche, L. (2000) Gender differences in pain. Journal of Orofacial Pain, 14, 169-184.

[32] Unell, L., Johansson, A., Carlsson, G.E., et al. (2006) Changes in reported orofacial symptoms over a ten-year period as reflected in two cohorts of fifty-year-old subjects. Acta Odontologica Scandinavica, 64, 202-208. doi: $10.1080 / 00016350500520276$

[33] Lund, I. and Lundeberg, T. (2008) Is it all about sex? Acupuncture for the treatment of pain from a biological and gender perspective. Acupuncture in Medicine, 26, 33-45. doi:10.1136/aim.26.1.33

[34] Dworkin, S.F., Huggins, K.H., Wilson, L., et al. (2002) A randomized clinical trial using research diagnostic criteria for temporomandibular disorders-axis II to target clinic cases for a tailored self-care TMD treatment program. Journal of Orofacial Pain, 16, 48-63.

[35] Öberg, T., Carlsson, G.E. and Fajers, C.M. (1971) The temporomandibular joint. A morphologic study on a human autopsy material. Acta Odontologica Scandinavica, 29, 349-384. doi:10.3109/00016357109026526

[36] Nakamura, K. (2006) Vitamin D and prevention of osteoporosis: Japanese perspective. Environmental Health and Preventive Medicine, 11, 271-276. doi:10.1007/BF02898016

[37] Von Korff, M., Dworkin, S.F. and Le Resche, L. (1990) Graded chronic pain status: An epidemiologic evaluation. Pain, 40, 279-291. doi:10.1016/0304-3959(90)91125-3

[38] Pincus, T. (2010) Are patient questionnaire scores as "scientific" as laboratory tests for rheumatology clinical care? Bull NYU Hospital for Joint Diseases, 68, 130-139.

[39] Hansson, T.L. (1992) Pathological aspects of arthritides and derangements. In: Sarnat, B.G. and Laskin, D.M, Eds., The Temporomandibular Joint: A Biological Basis for Clinical Practice, Saunders W.B. Company, Philadelpia, 165-182.

[40] Abdel-Fattah, R.A. (1992) Evaluating TMJ injuries. Wiley Law Publications, New York. 